\title{
ESTADO DEL ARTE DE LA INTERVENCIÓN CON FAMILIAS EN ATENCIÓN TEMPRANA DURANTE LA ÚLTIMA DÉCADA EN ESPAÑA
}

\author{
Estrella Fátima Rueda Aguilar \\ Departamento de Psicología Social (Universidad de Sevilla) \\ erueda@us.es
}

Recepción Artículo: 16 septiembre 2020

Admisión Evaluación: 21 octubre 2020

Informe Evaluador 1: 25 noviembre 2020

Informe Evaluador 2: 23 noviembre 2020

Aprobación Publicación: 27 noviembre 2020

\section{RESUMEN}

La intervención familiar supone un factor clave en la intervención de niños con cualquier tipo de discapacidad, por ello con este estudio se pretende revisar la literatura científica realizando una búsqueda bibliográfica que recoja los trabajos realizados sobre la familia en Atención Temprana. Se realizó una búsqueda sistemática en las bases de datos de Psicodoc y Psyclnfo de las publicaciones académicas realizadas en los últimos diez años (2010-2019) en España, con los siguientes descriptores: "Atención Temprana" y "Familia". Como resultado del proceso realizado se obtuvieron un total de 26 artículos. Los resultados presentados en este estudio permiten extraer diversas lecturas de interés tanto del contenido como de la productividad. Gran parte de los artículos revisados han intentado proporcionar una puesta al día de conceptos sobre la Atención Temprana, responder a preguntas sobre aspectos etiológicos, diagnósticos o terapéuticos, e incluso presentar casos clínicos con propuestas de intervenciones. Otra gran parte de los artículos analizados han sido estudios empíricos de diferentes metodologías cuyas temáticas han sido muy variadas, destacando los dedicados a los estilos de interacción entre los profesionales y las familias, las necesidades de formación y el desarrollo profesional de los trabajadores, la influencia de la discapacidad de un hijo en la familia, y la relación entre las interacciones de los progenitores con los hijos y el desarrollo adecuado infantil. En relación a la productividad, Ilama la atención que en la última década sólo se hallan encontrado 26 artículos de trabajo con familia contextualizados dentro de la Atencion Temprana en España. A pesar de la importancia del trabajo en familia resulta escasa la literatura que se centra en investigar sus prácticas.

Palabras claves: familia; atención temprana; trastornos del desarrollo; atención familiar; desarrollo en la infancia 


\title{
ESTADO DEL ARTE DE LA INTERVENCIÓN CON FAMILIAS EN ATENCIÓN TEMPRANA DURANTE LA ÚLTIMA DÉCADA EN ESPAÑA
}

\begin{abstract}
State of the art of intervention with families in early care during the last decade in spain. Family intervention is a key factor in the children development with any type of disability. Therefore, this study aims to review the scientific literature with a bibliographic search that includes articles about the family in early intervention. A systematic search was done in the Psicodoc and Psyclnfo databases of academic publications carried out in the last ten years (2010-2019) in Spain, with the following descriptors: "Early Intervention" and "Family". A total of 26 articles were obtained because of the process made. The results presented in this study allow different conclusions to be drawn about the content and productivity. Most of the reviewed articles have tried to provide an update of concepts about Early Intervention, answer questions about etiological, diagnostic or therapeutic aspects, and present clinical cases with proposed interventions. Other part of the analyzed articles have been empirical studies of different methodologies whose themes have been very varied, highlighting those dedicated to the styles of interaction between professionals and families, the training needs and professional development of workers, the influence of a child's disability on the family and the relationship between parental interactions with children and proper child development. Regarding productivity, in the last decade only 26 articles on family and Early Intervention in Spain have been found. Despite the importance of the family, there is little literature focused on investigating their practices.
\end{abstract}

Keywords: family; early intervention; developmental disabilities; family care; infant development

La aplicación de modelos teóricos psicológicos, como el ecológico y el transaccional, a ámbitos psicoeducativos y las aportaciones de la psicología social, hace que el niño sea visto como el resultado de un complejo proceso de interacciones entre el organismo y el ambiente. Hoy día es indiscutible la influencia que tienen variables como el estado emocional de la familia, los apoyos sociales o el estilo de crianza en el desarrollo del niño, y como diferentes evidencias han hecho que el concepto de Atención Temprana (AT en adelante) cambie de manera significativa.

La AT ha avanzado mucho en los últimos años, pasando de posiciones biologicistas a posiciones ambientalistas, y evolucionando hacia una integración de lo biológico, lo psicológico y lo social, que se manifiesta principalmente en la interacción del individuo con sus diferentes contextos y, por tanto en la necesidad de intervenir integralmente sobre todos estos factores en cada acción terapéutica.

Actualmente se asume de manera consensuada el concepto de AT según el Libro Blanco de la Atención Temprana (Grupo de Atención Temprana, 2005): "Se entiende por AT el conjunto de intervenciones dirigidas a la población infantil de 0-6 años, a la familia y al entorno, que tienen por objetivo dar respuesta lo más pronto posible a las necesidades transitorias o permanentes que presentan los niños con trastornos en su desarrollo o que tienen el riesgo de padecerlos. Estas intervenciones, que deben ser consideradas la globalidad del niño, han de ser planificadas por un equipo de profesionales de orientación interdisciplinar o transdisciplinar".

En esta definición se resalta la necesidad de contemplar un conjunto de actuaciones dirigidas a la población de 0 a 6 años pero también a la familia y a la comunidad. Según Candel (2005), la AT no debe entenderse como un tratamiento dirigido a un niño, sino como una serie de actuaciones dirigidas a niños, familias y a la comunidad en general. En esta misma línea, Gútiez, (2005) indica que el Grupo de Atención Temprana (GAT en adelante) describe un modelo de AT con una serie de características, entre las que se encuentra el protagonismo de la familia (figura 1). 
Figura 1. Características del modelo de AT descrito por el GAT. Fuente: Elaboración propia. Basado en Gútiez (2005)

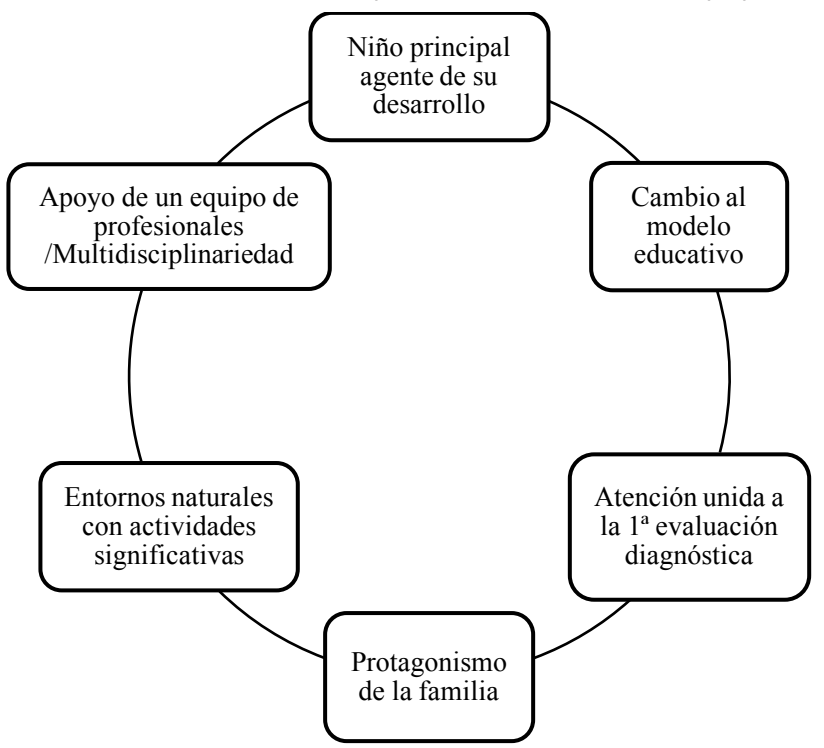

El principal objetivo de la AT es favorecer el desarrollo y el bienestar del niño y su familia, posibilitándole de forma más completa su integración en el medio familiar, escolar y social, así como su autonomía personal (Candel, 2005), es por ello que se trabajan áreas como son la cognitiva, autonomía, lenguaje o comunicación, y motora, además de asesorar, orientar e intervenir individual y/o grupalmente con las familias que tienen un hijo con cualquier discapacidad o riesgo de padecerla según los diferentes diagnósticos recogidos en la Organización Diagnóstica de la Atención Temprana (Federación Estatal de Asociaciones de Profesionales de Atención Temprana, 2008).

Las acciones encaminadas a la incorporación de prácticas centradas en la familia en AT han empezado a introducirse en España en los últimos años (Escorcia, García-Sánchez, Orcajada y Sánchez-López, 2016; GarcíaSánchez, Escorcia, Sánchez-López, Orcajada y Hernández-Pérez, 2014; Cañadas, 2014), como respuesta a las necesidades de mejora de la intervencion que se venían detectando (García-Sánchez et al., 2014; Gútiez, 2010; Gine, Gracia, Vilaseca y García-Die, 2006; García-Sánchez, 2002), tal y como recoge el Libro Blanco de AT (GAT, 2005).

Las actuales investigaciones en el área de la AT enfatizan la necesidad de avanzar hacia modelos de atención que permitan la participación de la familia. Los datos recabados evidenciaron la necesidad de hacer más de lo que se estaba haciendo al intervenir con la familia (Gine et al., 2006; Castellanos, García-Sánchez, Mendieta, Gómez y Rico, 2003; García-Sánchez, 2002), ya que las actuaciones más frecuentemente indicadas hacían referencia a intervenciones ambulatorias sobre el niño, y a un posicionamiento profesional de experto que orienta a la familia, y que no plantea una relación de colaboración, al mismo nivel, entre profesional y familia.

En 2011, el GAT constató la precariedad del tiempo dedicado a la atencion de la familia en los servicios ambulatorios existentes, ya que la estructura y configuración de estos equipos de intervencion estaban diseñadas para ofrecer una intervencion terapéutica ambulatoria dirigida fundamentalmente al niño, situación que en la que también influían las carencias formativas de los profesionales sobre la intervencion efectiva con familias a partir de prácticas basadas en la evidencia. 
Tanto organizaciones internacionales como la World Health Organization (WHO, 2012), la United Nations Educational, Scientific and Cultural Organization (UNESC0, 2015), la Division for Early Childhood of the Council for Exceptional Children (DEC, 2014), como asociaciones profesionales especializadas como la European Association on Early Childhood Intervention y la International Society on Early Intervention hicieron un Ilamamiento unánime para incorporar en la AT prácticas basadas en la evidencia y centradas en la familia que ayuden a mejorar la calidad de vida de las familias con niños con discapacidad.

La AT se ha desarrollado a partir de la década del 60, y desde entonces ha planteado diversos modelos según el rol que asumen los profesionales y la familia, así como el tipo de prácticas realizadas. García-Sánchez et al. (2014) sistematizan una clasificación de los modelos de AT a partir de la revisión de autores como Dunst, Johanson, Trivette y Hamby (1991) y Espe-Sherwindt (2008), y proponen 4 modelos: Modelo centrado en el profesional, Modelo familia-aliada, Modelo enfocado a la familia y Modelo centrado en la familia (tabla 1). Estos modelos representan el cambio en el estatus de los profesionales y las familias en el proceso de AT, observándose una evolución en los profesionales de expertos a colaboradores que se sitúan desde las necesidades y demandas de la familia, y como la familia asume un rol protagonista en el proceso de atención, se le reconocen sus capacidades y se fortalece la participación en la toma de decisiones según el aprendizaje y bienestar de su hijo.

Tabla 1. Modelos de intervención en AT

\begin{tabular}{|c|c|c|c|}
\hline \multicolumn{4}{|c|}{ MODELOS } \\
\hline $\begin{array}{l}\text { Centrado en el } \\
\text { profesional }\end{array}$ & Familia-aliada & $\begin{array}{l}\text { Enfocado a la } \\
\text { familia }\end{array}$ & $\begin{array}{l}\text { Centrado en la } \\
\text { familia }\end{array}$ \\
\hline La familia asume & profesionales & Los profesionales & Los profesionales \\
\hline un rol pasivo y los & identifican & consideran a las & consideran a las \\
\hline profesionales un & necesidades del niño & familias como & familias como \\
\hline $\begin{array}{l}\text { rol activo por } \\
\text { cuanto determinan }\end{array}$ & $\begin{array}{l}\text { y se considera que la } \\
\text { familia es capaz de }\end{array}$ & $\begin{array}{l}\text { consumidores que, } \\
\text { con asistencia, }\end{array}$ & $\begin{array}{l}\text { socios iguales o } \\
\text { colaboradores }\end{array}$ \\
\hline lo que necesita el & poner en práctica la & pueden elegir entre & necesarios para \\
\hline amilia, & interv & diversas & facilitar y mejorar \\
\hline así como la mejor & El profesional es & opciones que son & las posibilidades de \\
\hline forma de satisfacer & experto, quien decide & identificadas & el niño. \\
\hline $\begin{array}{l}\text { esas necesidades. } \\
\text { Los profesionales }\end{array}$ & y entrega & presentadas a la & La intervención es \\
\hline son considerados & pertinentes. & potencia que las & flexible y sensible a \\
\hline expertos, & familias & familias puedan & las necesidades de \\
\hline generando & convierten & elegir, para que & cada niño y de su \\
\hline relación & coterapeutas & experimenten & familia. \\
\hline dependencia de la & pseudoprofesionales, & independencia del & identificación \\
\hline & $\begin{array}{l}\text { que intentan re } \\
\text { en } \quad \text { casa }\end{array}$ & & $\begin{array}{l}\text { necesidades } \quad \text { se } \\
\text { realiza con ayuda }\end{array}$ \\
\hline & intervención & & de la propia familia. \\
\hline & $\begin{array}{l}\text { rehabilitadora que } \\
\text { haría un profesional. }\end{array}$ & & \\
\hline
\end{tabular}

Fuente: Elaboración propia. Basado en García et al. (2014)

En el metaanálisis realizado por Dunts, Triviette y Hamby (2008) sobre las prácticas llevadas a cabo con familias, se puso de manifiesto la presencia de dos indicadores del proceso de intervención y de su éxito en la práctica del profesional de AT: 
Relacionales, que incluyen comportamientos asociados con la buena práctica clínica (compasión, empatía y escucha activa, etc.) y con las funciones del profesional en la adquisición de competencias, fortalezas y capacidades de los miembros de la familia.

Participativos, que contemplan prácticas encaminadas a la fomentar la participación de los miembros de la familia en la toma de decisiones, el uso de sus habilidades y el desarrollo de nuevas habilidades para obtener los recursos deseados, y la colaboración con el profesional.

Desde hace unos años, España está viviendo un proceso de transformación gradual en la AT hacia un modelo centrado en la familia, incluso ya se puede empezar a vislumbrar un especial protagonismo en la intervención cada vez más centrada en la mejora de la calidad de vida de la familia en su conjunto, además de en el desarrollo del niño.

El trabajo con familias supone un factor clave en la intervención de niños con cualquier tipo de discapacidad, por ello con este estudio se pretende revisar la literatura científica realizando una búsqueda bibliográfica que recoja los trabajos realizados sobre la familia en AT en la última década en España.

\section{MÉTODO}

Para realizar este trabajo se utilizó el enfoque cualitativo descriptivo a través del método bibliográfico con el fin de indagar, recolectar, organizar, analizar e interpretar informaciones (Alfonso, 1995) en torno a la atención a la familia en AT. El propósito es la construcción de conocimientos y la sistematización objetiva y metódica de los estudios publicados con el objetivo de concretar el estado de la cuestión sobre el tema planteado (Sánchez-Meca, 2010). Para ello, se realizó una búsqueda sistemática en las bases de datos de Psicodoc y Psyclnfo de las publicaciones académicas realizadas en los últimos diez años (2010-2019) en España, con los siguientes descriptores: "Atención Temprana" y "Familia".

Los criterios de inclusión utilizados para la selección de los artículos fueron:

a. Tipo de fuente: Publicaciones académicas.

b. Publicados desde 2010 a 2019.

c. Centrado en algún aspecto de la intervención en AT con la familia.

d. Realizados en España.

A partir del número inicial de trabajos obtenidos ( $N=59)$, se hizo un primer cribado para seleccionar sólo las publicaciones académicas centradas en algún aspecto de la intervención en AT con la familia en España entre 2010 y 2019, resultando 26 artículos que fueron almacenado en el gestor bibliográfico Mendeley. Se extrajo la información de cada artículo elaborando una hoja de registro con los siguientes datos: año de realización del estudio, preguntas, hipótesis/objetivos, variables principales, método de análisis/ intervención y principales hallazgos. El registro de cada artículo se realizó por duplicado por dos juezas independientes. En los casos de discrepancias entre las investigadoras, se recurrió a un tercer juez cuando no consiguieron un acuerdo. En la figura 2 se presenta el diagrama de búsqueda. 
Figura 2. Diagrama de búsqueda

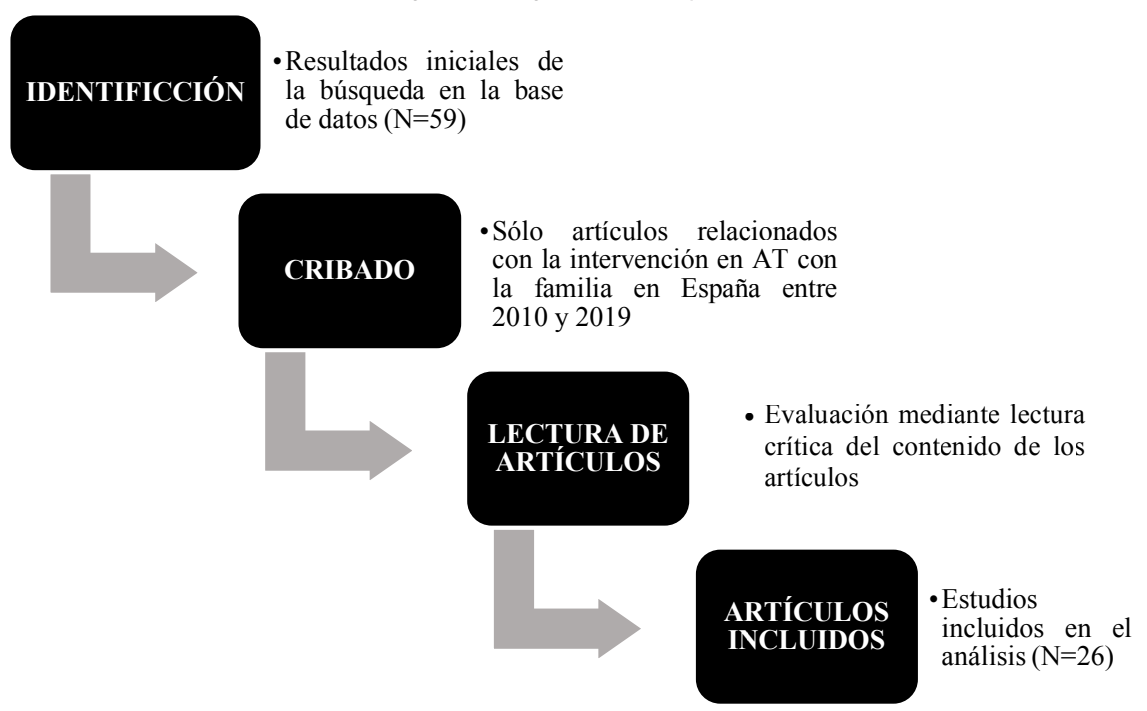

Se ha tenido en cuenta la guía y directrices propuestas por la declaración PRISMA, en aras de realizar un análisis que garantice sistematicidad y estandarización a lo largo de todo su proceso de realización (Urrutia y Bonfill, 2010).

\section{RESULTADOS}

Como resultado del proceso descrito, se obtuvieron un total de 26 artículos. En la figura 3 se presentan los artículos seleccionados clasificados según el año de publicación. El número de publicaciones por año aparecidas durante la última década en torno a los términos Atención Temprana y Familia ha registrado un ritmo de crecimiento disperso y poco constante, siendo los años 2015 y 2016 cuando se observa el mayor número de artículos anuales publicados $(\mathrm{N}=5)$.

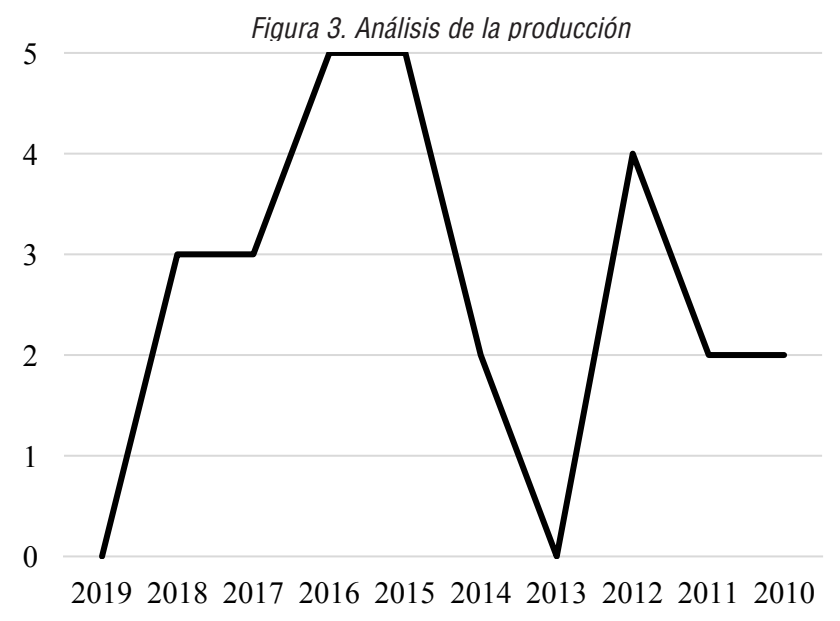


En el análisis de la producción por autores, se evidencia que un poco más de una tercera parte de los artículos analizados están realizados por tres autores, otra tercera parte por cuatro o más autores y tan sólo un 7.6\% están realizados por un autor (tabla 2).

\begin{tabular}{c|c}
\begin{tabular}{c} 
Tabla 2. Número de autores que participan en los artícul \\
\hline $\begin{array}{c}\text { Número de } \\
\text { autores }\end{array}$
\end{tabular} & $\begin{array}{c}\text { Número de } \\
\text { artículos }\end{array}$ \\
\hline 1 & 2 \\
2 & 5 \\
3 & 9 \\
4 & 4 \\
5 & 2 \\
6 & 1 \\
Más de 7 & 3 \\
\hline
\end{tabular}

Además, se observa un reparto desigual del número de artículos en que participaron los 73 autores. La distribución se ajustó a la Ley de Lotka (Lotka, 1926) según la cual la mayoría de los autores ( $N=62$ ) firman un solo trabajo, mientras que pocos participan en tres o más texto: dos firmas aparecieron en seis artículos (Claudia T. Escorcia-Mora; Francisco A. García Sánchez ), una firma apareció en cinco artículos (María Cristina Sánchez López) y dos firmas aparecieron en tres artículos (Margarita Cañada Pérez; Noelia Orcajada Sánchez). Estos datos se reflejan en la tabla 3.

Tabla 3. Distribución de las aportaciones por autor

\begin{tabular}{cl}
\hline $\begin{array}{c}\text { Número de } \\
\text { aportaciones }\end{array}$ & \multicolumn{1}{c}{ Autores } \\
\hline 6 & Claudia T. Escorcia-Mora; Francisco A. García Sánchez \\
5 & María Cristina Sánchez López \\
4 & --- \\
3 & Margarita Cañada Pérez; Noelia Orcajada Sánchez \\
& María Paz García-Martínez; Encarnación Hernández Pérez; Dolores \\
2 & Madrid-Vivar; Laura P. Perea Velasco; Julio Pérez López; David \\
& Sánchez Teruel \\
\hline
\end{tabular}

En cuanto a la producción por revistas, el reparto de los 26 publicaciones se distribuyó entre catorce revistas. La revista Anales de Psicología y la Revista de Logopedia, Foniatría y Audiología fueron las que más textos aportaron, en concreto cuatro textos cada una (tabla 4). 
Tabla 4. Producción por revista

\begin{tabular}{|c|c|}
\hline $\begin{array}{l}\text { Número } \\
\text { de } \\
\text { artículos }\end{array}$ & Revistas \\
\hline 4 & Anales de Psicología; Revista de Logopedia, Foniatría y Audiología \\
\hline 3 & Escritos de Psicología; \\
\hline 2 & $\begin{array}{l}\text { Revista de Psicología; Cuadernos de Psiquiatría y Psicoterapia del niño y } \\
\text { del adolescente; International Journal of Developmental and Educational } \\
\text { Psychology; Intersubjetivo: Revista de Psicoterapia Psicoanalítica y Salud } \\
\text { Bordón; Siglo Cero; Ciência \& Saúde Coletiva; Ansiedad y Estrés; }\end{array}$ \\
\hline 1 & $\begin{array}{l}\text { Universitas Psychologica; Revista Española sobre Discapacidad } \\
\text { Intelectual; Psicología Educativa }\end{array}$ \\
\hline
\end{tabular}

De los tipos de texto analizados, doce corresponden a estudios teóricos y catorce a estudios empíricos.

En los trabajos teóricos se han planteado diferentes revisiones bibliográficas de distinta tipología. Siguiendo la clasificación tradicional de los tipos de revisión bibliográfica (Icart y Canela 1994), los artículos planteados en este estudio se presentan en la tabla 5.

Tabla 5. Artículos analizados clasificados por tipos de revisión bibliográfica

\begin{tabular}{|c|c|c|}
\hline $\begin{array}{l}\text { Tipo de } \\
\text { revisión }\end{array}$ & $\mathrm{N}$ & Artículos \\
\hline Descriptiva & 5 & $\begin{array}{ll}\text { - } & \text { Dalmau, M.; Balcells-Balcells, A.; Giné, C.; Cañadas, M.; } \\
& \text { Casas, O.; Salat, Y.; Farré, V. y Calaf, N. (2017) } \\
\text { - } & \text { Escorcia Mora, C.T.; García Sánchez, F.A.; Orcajada Sánchez, } \\
& \text { N. y Sánchez López, M.C. (2016) } \\
\text { - } & \text { Espe-Sherwindt, M. y Serrano, A.M. (2016) } \\
\text { - } & \text { Martínez Moreno, A. y Calet, N. (2015) }\end{array}$ \\
\hline Evaluativa & 5 & $\begin{array}{ll}\text { - } & \text { Montagut Asunción, M.; D’Ocon Giménez, A. y Pastor } \\
& \text { Cerezuela, G. (2017) } \\
\text { - } & \text { Roncallo, C.P.; Sánchez de Miguel, M. y Arranz Freijo, } \\
& \text { E.(2015) } \\
\text { - Camarero, B.; Cuéllar-Flores, I.; Eimil, B.; Palacios, I.; Río, } & \text { T. y del García-Villanova, F. (2012) } \\
\text { - } & \text { Torres Gómez, A. y Rabasot Solán, M.A. (2011) } \\
\text { - } & \text { González Serrano, F. (2010) }\end{array}$ \\
\hline $\begin{array}{l}\text { Casos } \\
\text { clínicos con } \\
\text { revisión }\end{array}$ & 2 & $\begin{array}{ll}\text { - } & \text { Molina Vizcaíno, J.R. (2014) } \\
\text { - } & \text { Gracia, M.; Ausejo, R. y Porras, M. (2010) }\end{array}$ \\
\hline
\end{tabular}

Los cinco artículos que presentan una revisión bibliográfica descriptiva proporcionan una puesta al día de conceptos de la AT en constante evolución, así encontramos que

- Dalmau et al. (2017) ofrecen una propuesta metodológica para la implementación del modelo centrado en la familia en los centros de AT de nuestro país.

- Escorcia Mora et al. (2016) delimitan el concepto de prácticas centradas en la familia en AT, repasando los elementos claves que la han impulsado desde su aparición y los componentes que la caracterizan y diferencian de otras prácticas, y resaltan la importancia del logopeda en los equipos transdisciplinares de AT. 
- Espe-Sherwindt y Serrano (2016) describen evidencias de las prácticas centradas en la familia, cómo y por qué se han convertido en un aspecto clave para la intervención en la comunicación en la primera infancia, para ello definen las características que definen y componen las prácticas centradas en la familia.

- Martínez Moreno y Calet (2015) analizan la evolución que ha tenido la AT, examinan la transformación que ha sufrido el papel de la familia, revisan los estudios más relevantes en el área y proponen nuevas líneas de actuación.

- García-Sánchez, Escorcia y Sánchez-López. (2014) reflexionan sobre el cambio de paradigma en AT de un modelo de tratamiento ambulatorio a prácticas centradas en la familia, desmitificando los conflictos que se generan en el pensamiento del profesional al plantearse el cambio de enfoque y describiendo las necesidades de formación que podrían facilitar el cambio de paradigma.

Por otro lado, se realizaron cinco revisiones bibliográficas evaluativas para responder a preguntas específicas sobre aspectos etiológicos, diagnósticos, clínicos o terapéuticos, para ello:

- Montagut Asunción, D’Ocon Giménez. y Pastor Cerezuela (2017) exploran la presencia en los hermanos de niños con trastorno del espectro autista de conductas de atención conjunta, comunicación y percepción gestual, rendimiento en tareas lingüísticas, percepción de rostros y de otros estímulos sociales, sonrisa social y presencia de juego simbólico, y lo comparan con hermanos de niños con desarrollo típico.

- Roncallo, Sánchez de Miguel y Arranz Freijo (2015) analizan la importancia del vínculo materno-fetal en el desarrollo posterior del bebe y en la promoción de la salud materno-infantil, y sus implicaciones en la AT.

- Camarero et al. (2012) definen el impacto emocional en las familias, en especial en las madres, con el nacimiento de un hijo prematuro, para ello describen como el estrés y la desorganización emocional de los progenitores interfieren en el desarrollo psicológico y emocional del niño prematuro, y plantean una intervención dirigida a los problemas de vinculación bebé-progenitores.

- Torres Gómez y Rabasot Solán (2011) examinan las consecuencias emocionales que tiene un diagnóstico de discapacidad para las familias y los hijos en función de las características de la familia y de la situación emocional en la que se encuentran los padres y madres en el momento del diagnóstico

- González Serrano (2010) aborda las situaciones de gran prematuridad, características del bebé prematuro y las relaciones tempranas con el entorno y la familia, planteando propuestas de atención y acompañamiento a los bebés prematuros y sus familias, en el medio hospitalario y tras el alta, para prevenir dificultades futuras en la relación con el entorno cercano y el apego, y en el desarrollo cognitivo, emocional y social del niño.

Respecto a los textos de casos clínicos con revisión, se han analizado dos artículos que realizan una exposición de un caso clínico con sus propuestas de intervenciones terapéuticas, para tal fin:

- Molina Vizcaíno (2014) reflexiona desde el paradigma hermenéutico sobre la utilidad psicoterapéutica del uso, en los centros de AT del Children Behavior CheckList (CBCL) 11/2- 5 para preescolares en el trabajo con familia y expone un caso clínico para ilustrar la utilidad de este instrumento.

- Gracia, Ausejo y Porras (2010) presentan una breve revisión de las tendencias actuales en la intervención temprana en lenguaje y comunicación y examinan dos casos que evidencian que en nuestro país algunos logopedas están trabajando con modelos que incluyen a las familias y maestras con el fin de ayudarles a incorporar estrategias que promuevan el desarrollo de sus capacidades comunicativas y lingüísticas.

Por otro lado, los catorce trabajos empíricos revisados han sido estudios realizados con distintas metodologías (tabla 6). 
Tabla 6. Clasificación artículos empíricos en función de la metodología de estudio

\begin{tabular}{|c|c|c|}
\hline Metodología & $\mathrm{N}$ & Artículos \\
\hline $\begin{array}{l}\text { Estudio } \\
\text { transversal }\end{array}$ & 5 & $\begin{array}{l}\text { - } \quad \text { García-Sánchez, F.A.; Rubio-Gómez, N.; Orcajada-Sánchez, } \\
\text { N.; Escorcia-Mora, C.T. y Cañadas, M. (2018) } \\
\text { - Escorcia-Mora, C.T.; García-Sánchez, F.A., Sánchez-López, } \\
\text { M.C., Orcajada, N. y Hernández-Pérez, E. (2018) } \\
\text { - Marco Arenas, M.; Sánchez López, M.C. y García Sánchez, } \\
\text { F.A. (2018) } \\
\text { - Pérez-López, J.; Pérez-Lag, M.; Montealegre, M.P. y Perea } \\
\text { Velasco L.P. (2012) } \\
\text { - Perea Velasco, L.P.; Martínez-Fuentes, M.T.; Pérez-López, J. y } \\
\text { Díaz-H., A. (2011) }\end{array}$ \\
\hline $\begin{array}{l}\text { Estudio de } \\
\text { caso }\end{array}$ & 2 & $\begin{array}{l}\text { - Cañada Pérez, M.; Escorcia Mora, C.T.; Martínez Rico, G.; } \\
\text { Alonso Martín, M.; Ayuso Blázquez, V.; Domenech Varea, M.; } \\
\text { Franco Castellano, I.; Moltó Soler, E.; Roca Hurtuna, M. } \\
\text { Santandreu García, A. y Sanchís Martínez, C. (2016) } \\
\text { - Robles Bello, M.; Sánchez-Teruel, D. y Romero-Prado, A.B. } \\
\text { (2015) }\end{array}$ \\
\hline $\begin{array}{l}\text { Estudio } \\
\text { instrumental }\end{array}$ & 1 & $\begin{array}{l}\text { - Escorcia Mora, C.T.; García Sánchez, F.A.; Sánchez López, } \\
\text { M.C. y Hernández Pérez, E. (2016) }\end{array}$ \\
\hline $\begin{array}{l}\text { Estudio } \\
\text { cualitativo }\end{array}$ & 2 & $\begin{array}{l}\text { - García, M.P.; Madrid, D. y Galante, R. (2017) } \\
\text { - Clarissa Altina, C.; Paz-Lourido, B. y Verger Gelabert, S. } \\
\text { (2016) }\end{array}$ \\
\hline $\begin{array}{l}\text { Estudio } \\
\text { cuasi- } \\
\text { experimental }\end{array}$ & 1 & - $\quad$ Sánchez, D. y Roble, M.A. (2015) \\
\hline $\begin{array}{l}\text { Estudio } \\
\text { descriptivo }\end{array}$ & 2 & $\begin{array}{ll}\text { - } & \text { Ruiz-Fernández, I.; Fajardo, I.; Bermejo, L.; Calzado, Z; Pérez- } \\
& \text { Ruiz, M.; García, V. y Gordillo, M. (2012) } \\
\text { - } & \text { Mayorga-Fernández, M.J.; Madrid-Vivar, D. y García- } \\
& \text { Martínez, M.P. (2015) }\end{array}$ \\
\hline $\begin{array}{l}\text { Estudio ex } \\
\text { post facto }\end{array}$ & & de Andrés Viloria, C. y Guinea, C.L. (2012) \\
\hline
\end{tabular}

De los trabajos revisados se han evidenciado cinco estudios transversales en los que los distintos autores plantean distintos objetivos:

- Escorcia-Mora et al. (2018) tratan de profundizar en cómo se están desarrollando las prácticas profesionales e identificar posibilidades de mejora.

- García-Sánchez et al. (2018) analizan los aspectos que los profesionales consideran más importantes y necesarios para su formación en prácticas centradas en la familia.

- Marco Arenas, Sánchez López y García Sánchez (2018) comprueban si determinadas habilidades de inteligencia emocional de los profesionales de AT están vinculadas con un mayor o menor ejercicio de prácticas relacionales en las interacciones con familia.

- Pérez-López et al. (2012) estudian si existen relaciones entre el estrés experimentado por los padres y madres debido a la crianza de sus hijos y el desarrollo cognitivo, comunicativo y motor en los niños.

- Perea Velasco et al. (2011) investigan en profundidad las características de la interacción madre-hijo para indagar en el impacto que tiene en el desarrollo temprano de los niños, para ello analizaron la relación entre la 
sensibilidad materna y la calidad de la interacción infantil, y como ésta última afecta el desarrollo mental en su primer año de vida.

Los dos estudios de casos estuvieron dedicados a:

- Cañada Pérez et al. (2016) muestran evidencias de los aspectos más relevantes de las prácticas centradas en la familia y en entornos naturales a través de un caso clínico.

- Robles Bello, Sánchez-Teruel y Romero-Prado, (2015) evalúan el nivel de estrés y las relaciones de pareja en las madres de dos niñas (trisomía 22 y trisomía 21), así como su perfil evolutivo, antes y después de la aplicación de un tratamiento.

Respecto a los dos estudios descriptivos.

- Ruiz-Fernández et al. (2012) plantearon conocer las necesidades de las familias que acuden a un Centro de Atención e Intervención Temprana generalista, con la finalidad de diseñar un programa que responda a las mismas.

- Mayorga-Fernández, Madrid-Vivar, y García-Martínez (2015) propusieron conocer el grado de información que las familias de niños con acondroplasia tienen en el momento del nacimiento de su hijo, de qué forma acceden a los servicios de AT, valorando las dificultades de la entrada su implicación en los mismos, y plantear la necesidad de una guía de AT en acondroplasia, que ayude en todo el proceso.

Los dos estudios cualitativos se centraron en:

- García, Madrid. y Galante (2017) examinar y comprender el funcionamiento familiar en lo que respecta a las emociones, sentimientos, necesidades y dificultades cuando un niño con algún tipo de diversidad funcional, necesitaba la ayuda de un sistema aumentativo 0 alternativo de comunicación.

- Clarissa Altina, Paz-Lourido y Verger Gelabert. (2016) indagar en la influencia de los apoyos en las familias que tienen un hijo con discapacidad, con el fin de identificar en qué aspectos pueden las políticas públicas influenciar una mejora en la calidad de vida familiar.

Por último, en el estudio instrumental de Escorcia Mora et al. (2016) se presenta el Cuestionario de Estilos de Interacción entre Padres y Profesionales en Intervención Temprana, en el estudio cuasiexperimental Sánchez y Roble (2015) se valora si el nivel de estrés en padres de niños con Síndrome de Down mejora tras la realización de un programa de entrenamiento en resiliencia y en el estudio ex post facto de Andrés Viloria y Guinea (2012) se aporta la reflexión y los resultados de dos estudios sobre la repercusión del nivel de estrés en las familias en las que hay un miembro con deficiencia intelectual y la organización, planificación y recursos de la intervención familiar que realizan los profesionales implicados en los ámbitos de actuación en AT.

En la tabla 7 se describen distintas características de los catorce estudios empíricos analizados. 
Tabla 7. Análisis de los estudios empírico

\begin{tabular}{|c|c|c|c|}
\hline $\begin{array}{c}\text { AUTORE } \\
\text { S } \\
\end{array}$ & MUESTRA & $\begin{array}{c}\text { VARIABLES/ } \\
\text { INSTRUMENTOS }\end{array}$ & CONCLUSIONES \\
\hline $\begin{array}{l}\text { García- } \\
\text { Sánchez et } \\
\text { al. (2018) }\end{array}$ & $\begin{array}{c}121 \\
\text { profesionale } \\
\mathrm{s}\end{array}$ & $\begin{array}{l}\text { Necesidad de formación } \\
\text { percibida, desarrollo } \\
\text { profesional, inteligencia } \\
\text { emocional, prácticas } \\
\text { relacionales y } \\
\text { características de los } \\
\text { participantes y de su } \\
\text { trabajo } \\
\text { / Cuestionario ad hoc }\end{array}$ & $\begin{array}{l}\text { Se han identificado cuatro } \\
\text { bloques de acciones formativas: } \\
\text { 1. Herramientas y técnicas. } \\
\text { 2. Transdisciplinariedad. } \\
\text { 3. Herramientas y estrategias } \\
\text { de prácticas centradas en } \\
\text { familias. } \\
\text { 4. Filosofía del modelo. }\end{array}$ \\
\hline $\begin{array}{l}\text { Escorcia- } \\
\text { Mora et al. } \\
(2018)\end{array}$ & $\begin{array}{c}504 \text { familias } \\
\text { y } 187 \\
\text { profesionale } \\
\text { s }\end{array}$ & $\begin{array}{l}\text { Prácticas relacionales y } \\
\text { prácticas participativas } \\
\text { / Cuestionario de } \\
\text { Estilos de Interacción } \\
\text { entre Padres y } \\
\text { Profesionales en AT }\end{array}$ & $\begin{array}{l}\text { Los profesionales desarrollan } \\
\text { más prácticas relacionales y } \\
\text { menos prácticas participativas } \\
\text { con las familias; y creen que } \\
\text { hacen más prácticas } \\
\text { participativas de las que las } \\
\text { familias realmente perciben. }\end{array}$ \\
\hline $\begin{array}{l}\text { Marco } \\
\text { Arenas, } \\
\text { Sánchez } \\
\text { López y } \\
\text { García } \\
\text { Sánchez } \\
(2018)\end{array}$ & & $\begin{array}{c}\text { Habilidades de } \\
\text { inteligencia emocional } \\
\text { y prácticas relacionales } \\
/ / \\
\text { Inventario sobre } \\
\text { Práctica Profesional en } \\
\text { AT e Inteligencia } \\
\text { Emocional: Trait } \\
\text { MetaMood Scale } \\
\end{array}$ & $\begin{array}{l}\text { Los profesionales de AT } \\
\text { realizan prácticas relacionales } \\
\text { en sus intervenciones y poseen } \\
\text { altos niveles de habilidades } \\
\text { emocionales. Se hallan } \\
\text { diferencias significativas en las } \\
\text { prácticas relacionales en } \\
\text { función de las habilidades de } \\
\text { inteligencia emocional. }\end{array}$ \\
\hline $\begin{array}{l}\text { García, } \\
\text { M.P.; } \\
\text { Madrid, D. } \\
\text { y Galante, } \\
\text { R. (2017) }\end{array}$ & $\begin{array}{l}8 \text { menores, } \\
\text { sus familias } \\
\text { y } 11 \\
\text { profesionale } \\
\text { s de AT }\end{array}$ & $\begin{array}{l}\text { Sistema aumentativo o } \\
\text { alternativo de } \\
\text { comunicación y } \\
\text { funcionamiento familiar } \\
\text { / Grupos focales }\end{array}$ & $\begin{array}{l}\text { Se enfatizan las emociones y } \\
\text { sentimientos que surgen en los } \\
\text { entornos familiares cuando a la } \\
\text { diversidad funcional se suma } \\
\text { las dificultades para la } \\
\text { comunicación en los diferentes } \\
\text { entornos. } \\
\text { Los profesionales expresan la } \\
\text { importancia de conocer las } \\
\text { necesidades de los menores y } \\
\text { sus familias para colaborar en la } \\
\text { búsqueda de soluciones. }\end{array}$ \\
\hline $\begin{array}{l}\text { Cañada } \\
\text { Pérez et } \\
\text { al.(2016) }\end{array}$ & 1 mina & $\begin{array}{c}\text { Desarrollo evolutivo, } \\
\text { perfil funcional de } \\
\text { fortalezas y contexto } \\
\text { familiar y escolar } \\
\text { / Inventario de } \\
\text { Desarrollo de Battelle, } \\
\text { Escala de Participación, } \\
\text { Autonomía y } \\
\text { Relaciones Sociales de }\end{array}$ & $\begin{array}{l}\text { Mejoría en el desarrollo } \\
\text { funcional de la pequeña y tanto } \\
\text { la familia como la profesora se } \\
\text { sienten más competentes, } \\
\text { empoderados y con mejor } \\
\text { calidad de vida. Se refleja la } \\
\text { importancia del trabajo } \\
\text { transdisciplinar, la colaboración } \\
\text { entre diferentes agentes sociales }\end{array}$ \\
\hline
\end{tabular}




\begin{tabular}{|c|c|c|c|}
\hline & & $\begin{array}{c}\text { McWilliam e } \\
\text { Younggren, Entrevista } \\
\text { basadas en rutinas y } \\
\text { Ecomapa }\end{array}$ & $\begin{array}{l}\text { y el indiscutible papel de la } \\
\text { familia. }\end{array}$ \\
\hline $\begin{array}{l}\text { Escorcia } \\
\text { Mora et al. } \\
(2016)\end{array}$ & $\begin{array}{c}4 \text { Grupos. } \\
\text { Focal } \\
\text { familias:13 } \\
\text { padres, } \\
\text { Enfoque } \\
\text { profesionale } \\
\text { s: } 15, \text { Juicio } \\
\text { experto: } 6 \\
\text { psicólogos, } \\
5 \\
\text { educadores } \\
\text { y } 5 \text { madres } \\
\text { y } \\
\text { Profesionale }\end{array}$ & $\begin{array}{c}\text { Prácticas relacionales y } \\
\text { participativas y estilos } \\
\text { de interacción de los } \\
\text { profesionales con las } \\
\text { familias } \\
\text { / Cuestionario de } \\
\text { Estilos de Interacción } \\
\text { entre Padres y } \\
\text { Profesionales (versión } \\
\text { profesionales y } \\
\text { cuidadores) }\end{array}$ & $\begin{array}{l}\text { El Cuestionario de Estilos de } \\
\text { Interacción entre Padres y } \\
\text { Profesionales: puede identificar } \\
\text { estilos de interacción y } \\
\text { prácticas relacionales y } \\
\text { participativas y } \\
\text { profesionales y familias en la } \\
\text { intervención temprana. Es una } \\
\text { herramienta útil para } \\
\text { investigaciones } \\
\text { globalizadora sobre los } \\
\text { servicios de intervención } \\
\text { temprana, y para analizar las } \\
\text { fortalezas y debilidades de los } \\
\text { servicios. }\end{array}$ \\
\hline $\begin{array}{l}\text { Clarissa } \\
\text { Altina, } \\
\text { Paz- } \\
\text { Lourido y } \\
\text { Verger } \\
(2016)\end{array}$ & $\begin{array}{l}4 \text { padr } \\
16 \text { mac } \\
\text { de nir̃ }\end{array}$ & $\begin{array}{c}\text { Tipos de apoyos, } \\
\text { políticas públicas y } \\
\text { calidad de vida familiar } \\
\text { / Entrevista } \\
\text { semiestructurada }\end{array}$ & $\begin{array}{l}\text { Importancia de los apoyos } \\
\text { familiares y de las redes de } \\
\text { padres de niños con } \\
\text { discapacidad. } \\
\text { Destaca el papel de los apoyos } \\
\text { profesionales y cómo los } \\
\text { recortes en servicios públicos } \\
\text { de tipo sanitario y social han } \\
\text { agudizado una inequidad en } \\
\text { salud de estas familias. }\end{array}$ \\
\hline $\begin{array}{l}\text { Mayorga- } \\
\text { Fernández, } \\
\text { Madrid- } \\
\text { Vivar y } \\
\text { García- } \\
\text { Martz } \\
(2015)\end{array}$ & 58 familias & $\begin{array}{l}\text { Participación familiar } \\
\text { en la toma de } \\
\text { decisiones y elección de } \\
\text { un coordinador asesor, } \\
\text { apoyo emocional, } \\
\text { social, informativo y } \\
\text { formativo y orientación } \\
\text { sobre apoyos } \\
\text { económicos, sanitarios, } \\
\text { educativos y sociales } \\
\text { / Cuestionario de } \\
\text { satisfacción ad hoc }\end{array}$ & $\begin{array}{l}\text { Un } 55.70 \% \text { de las familias } \\
\text { afirman que la entidad de AT } \\
\text { tendría que facilitar espacios } \\
\text { para el intercambio, y para un } \\
67.24 \% \text { estos espacios tendrían } \\
\text { que ser permanentes. Poner en } \\
\text { marcha un programa de apoyo } \\
\text { para familias puede ser más } \\
\text { fácil desde un CAIT para } \\
\text { facilitar a las familias los } \\
\text { apoyos necesarios, } \\
\text { brindándoles espacio } \\
\text { emocional y de comunicación } \\
\text { entre familias con más } \\
\text { experiencia. }\end{array}$ \\
\hline $\begin{array}{l}\text { Robles } \\
\text { Bello, } \\
\text { Sánchez- } \\
\text { Teruel } \\
\end{array}$ & 2 madres & $\begin{array}{l}\text { Desarrollo infantil, } \\
\text { estrés parental y ajuste } \\
\text { en la pareja }\end{array}$ & $\begin{array}{l}\text { Se obtuvo un perfil específico } \\
\text { del niño con trisomía 22, } \\
\text { caracterizado por un desarrollo } \\
\text { evolutivo atípico en diferentes }\end{array}$ \\
\hline
\end{tabular}




\begin{tabular}{|c|c|c|}
\hline $\begin{array}{l}\text { y Romero- } \\
\text { Prado } \\
(2015)\end{array}$ & & $\begin{array}{l}\text { / Escala Brunet-Lézine } \\
\text { Revisada, Índice de } \\
\text { Estrés Parental y Escala } \\
\text { de Ajuste Diádico-EAD }\end{array}$ \\
\hline $\begin{array}{l}\text { Sánchez y } \\
\text { Roble } \\
(2015)\end{array}$ & $\begin{array}{l}2 \text { Grupos. } \\
\text { Experiment } \\
\text { al: } 19 \\
\text { padres y } 20 \\
\text { madres y } \\
\text { Control: } 18 \\
\text { padres y } 20 \\
\text { madres }\end{array}$ & $\begin{array}{c}\text { Resiliencia y estrés } \\
\text { / Hojas de datos } \\
\text { sociodemográficos e } \\
\text { Índice de estrés parental } \\
\text { versión reducida }\end{array}$ \\
\hline
\end{tabular}

\begin{tabular}{lcc}
\hline & & \\
& & \\
& & \\
de Andrés & 250 & \\
$\begin{array}{l}\text { Viloria } \\
\text { Guinea }\end{array}$ & profesionale & $\begin{array}{l}\text { Estrés familiar } \\
\text { / Observación }\end{array}$
\end{tabular}

\begin{abstract}
áreas. $\mathrm{Se}$ encontraron diferencias en los niveles de estrés y en la dinámica de la relación de pareja entre las madres de los niños evaluados. Se considera la necesidad de implementar programas de intervención temprana diferenciados y específicos.
\end{abstract}

Reducción de estrés parental del cuidado del niño y del estrés tras la aplicación del programa. Importante aplicar tratamientos para el fomento de la resiliencia en padres que amplifiquen los efectos positivos del proceso de atención infantil temprana en niños con Síndrome de Down y sus familias.

La relación con los padres es un elemento básico, ya que cualquier tipo de intervención que se realice con ellos repercutirá positiva $\mathrm{o}$ negativamente en el niño con discapacidad y en el resto de los miembro de la familia. El estrés es un factor que aparecen en las familias, más en madres que en padres, por lo que es un aspecto s considerar en la planificación de la intervención.

\begin{tabular}{|c|c|c|c|}
\hline $\begin{array}{l}\text { Pérez- } \\
\text { López et } \\
\text { al. (2012) }\end{array}$ & $\begin{array}{l}18 \text { niños, } \\
\text { sus padres y } \\
\text { sus madres }\end{array}$ & 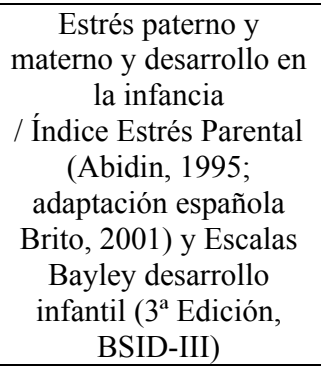 & $\begin{array}{l}\text { Las madres puntúan más alto } \\
\text { que los padres en las diferentes } \\
\text { dimensiones de estrés, aunque } \\
\text { sólo se encuentran diferencias } \\
\text { significativas entre el malestar } \\
\text { paterno de los progenitores y el } \\
\text { desarrollo motor de los niños } \\
\text { que se puede ver afectado por el } \\
\text { estrés de las madres. }\end{array}$ \\
\hline $\begin{array}{l}\text { Ruiz- } \\
\text { Fernández } \\
\text { et al. } \\
(2012)\end{array}$ & 17 familias & $\begin{array}{l}\text { Conocimiento y } \\
\text { aceptación de la } \\
\text { discapacidad: } \\
\text { Acondroplasia } \\
\text { / Cuestionario ah hoc }\end{array}$ & $\begin{array}{l}\text { El grado de conocimiento de los } \\
\text { padres sobre la discapacidad, en } \\
\text { el momento del nacimiento, es } \\
\text { muy escaso, siendo la } \\
\text { información mayoritariamente } \\
\text { de tipo médico. Las madres se }\end{array}$ \\
\hline
\end{tabular}

Estrés paterno y y desarrollo en dice Estrés Parental (Abidin, 1995; adaptación española Brito, 2001) y Escalas Bayley desarrollo BSID-III) Conocimiento y aceptación de la discapacidad: Acondroplasia
Cuestionario ah hoc 


\begin{tabular}{|c|c|c|c|}
\hline & & & $\begin{array}{l}\text { implican de una forma activa en } \\
\text { la intervención, aunque no } \\
\text { existe una buena aceptación de } \\
\text { la acondroplasia en estos } \\
\text { primeros años. }\end{array}$ \\
\hline $\begin{array}{l}\text { Perea } \\
\text { Velasco et } \\
\text { al (2011) }\end{array}$ & $\begin{array}{l}19 \text { niños }(11 \\
\text { niños y } 8 \\
\text { niñas) y sus } \\
\text { respectivas } \\
\text { madres }\end{array}$ & $\begin{array}{c}\text { Interacción madre-hijo, } \\
\text { sensibilidad materna, } \\
\text { apego, alteraciones del } \\
\text { desarrollo, ajuste } \\
\text { psicológico y calidad de } \\
\text { vida } \\
\text { / Escalas Bayley de } \\
\text { Desarrollo Infantil } \\
\text { (BSID-II; Bayley, } \\
\text { 1993) y CARE-Index, } \\
\text { Índice experimental de } \\
\text { relación niño-adulto de } \\
\text { Crittenden (2001) }\end{array}$ & $\begin{array}{l}\text { La sensibilidad materna no es } \\
\text { una característica individual, } \\
\text { sino un factor que surge de la } \\
\text { dinámica de las interacciones } \\
\text { madre-hijo. Está relacionada } \\
\text { significativamente con el } \\
\text { comportamiento cooperativo en } \\
\text { el niño. Ambos aspectos } \\
\text { promueven una interacción } \\
\text { sincrónica y de calidad que se } \\
\text { relaciona positivamente con } \\
\text { mayores índices de desarrollo } \\
\text { mental. }\end{array}$ \\
\hline
\end{tabular}

Por último, para el análisis de los contenidos de todos los textos analizados en este estudio se creó un sistema de categoría a partir de las variables estudiadas, resultando cinco grupos principales de contenidos (figura 5):

Estilos de interacción de los profesionales con las familias: prácticas relacionales y participativas.

Formación y competencias profesionales: necesidad de formación percibida, desarrollo profesional e inteligencia emocional.

Estrés y discapacidad: ajuste en la pareja, resiliencia, consecuencias en la familia,...

Prácticas centradas en las familias: tipos de apoyos, participación familiar, apoyo emocional, social, informativo y formativo, orientación, empoderamiento, conocimiento y aceptación de la discapacidad, calidad de vida, ...

Relaciones progenitores-hijos/as y desarrollo infantil: interacciones madre-hijo, sensibilidad materna, apego, relaciones tempranas, crianza, vínculo materno-fetal, vínculo postnatal madre-bebe, salud materno-infantil, ...

\section{DISCUSIÓN}

El papel de la familia en la educación y el adecuado desarrollo del niño es fundamental. Las responsabilidades parentales durante la etapa de la infancia son amplias e intensas, y están determinadas por todos los cuidados físicos, psicológicos y sociales que tienen que dispensar y mantener, y en definitiva por la conservación del bienestar del menor. Es por ello, que los padres y madres se convierten en parte esencial de un sistema, donde cualquier intervención que se realice sobre ellos repercutirá en el niño con discapacidad y en el resto de los miembros de la familia.

La participación de la familia en el ámbito de la AT en España ya es una realidad, No existe ninguna duda de que la familia tiene un papel de gran relevancia en las intervenciones que se implementan en estos servicios. En este sentido, los profesionales manifiestan un alto consenso sobre lo fundamental que resulta la implicación de la familia para la consecución de una mayor eficacia en el tratamiento, y por tanto, en el desarrollo del niño. 


\section{ESTADO DEL ARTE DE LA INTERVENCIÓN CON FAMILIAS EN ATENCIÓN TEMPRANA DURANTE \\ LA ÚLTIMA DÉCADA EN ESPAÑA}

Figura 4. Mapa conceptual AT y familia

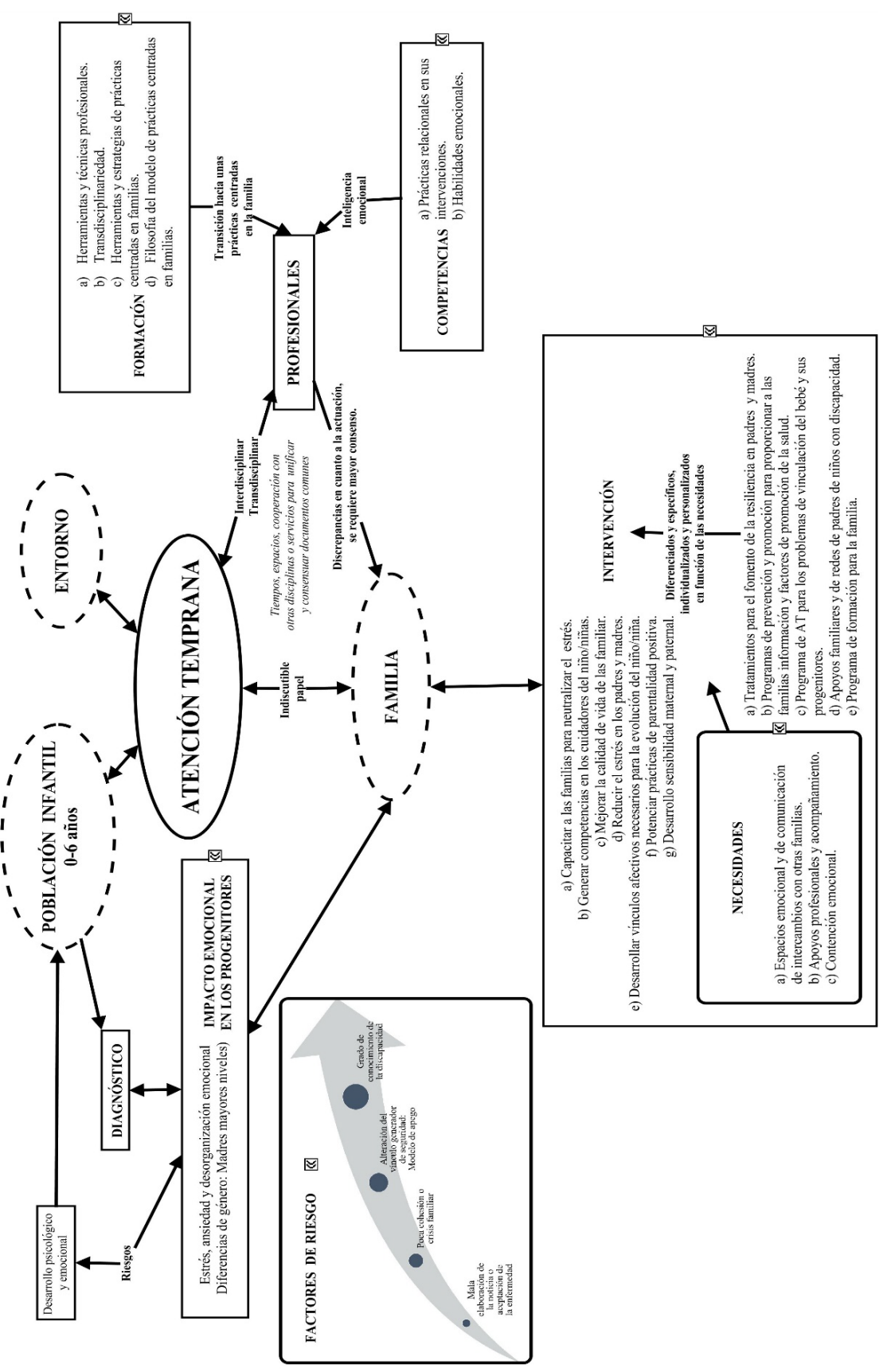


En AT existe un gran acuerdo sobre que el desarrollo cognitivo infantil se ve facilitado por la calidad de las interacciones familiares y está mediado por la relación de los niños con sus progenitores-cuidadores. Teniendo en cuenta esta premisa, es fundamental que para evaluar e intervenir se incluyan las características de cada contexto familiar, ya que este abordaje facilitará la definición de los objetivos de intervención y ayudará a prevenir alteraciones en el desarrollo infantil, además de potenciar la promoción de un desarrollo integral. Por lo tanto, es imprescindible que los profesionales de la AT conozcan la situación de cada uno de los componentes del sistema familiar y sepan dar respuestas a las necesidades específicas y las demandas concretas de cada familia.

Consecuentemente se hace necesario continuar sensibilizando a los profesionales de la AT para que sigan considerando a la familia como una parte activa y principal de la intervención, ya que en ocasiones la realidad del trabajo diario en AT se ve afectado por multitud de factores (faltas de recursos, escasez de tiempo, cansancio, sobrecarga de trabajo, ...) que pueden favorecer que la tendencia ya superada y en desuso de centrar la intervención exclusivamente sobre el niño. En palabras de Brown, Galambos, Poston y Turnbull (2007): "Ios profesionales deben enriquecer a las familias, proporcionándoles recursos para poder tomar decisiones satisfactorias, para mejorar la calidad de vida no solo de su hijo con discapacidad o con dificultades en el desarrollo, sino también la calidad de vida de la familia en su conjunto".

Los resultados presentados en este estudio permiten extraer diversas lecturas de interés tanto del contenido como de la productividad de los estudios llevados a cabo bajo los términos AT y familia en la última década en España.

Con respecto al contenido, gran parte de los artículos revisados han intentado proporcionar una puesta al día de conceptos sobre la AT, responder a preguntas sobre aspectos etiológicos, diagnósticos o terapéuticos, e incluso presentar casos clínicos con propuestas de intervenciones. Otra gran parte de los artículos analizados han sido estudios empíricos de diferentes metodologías cuyas temáticas han sido muy variadas, destacando los dedicados a los estilos de interacción entre los profesionales y las familias, las necesidades de formación y el desarrollo profesional de los trabajadores de la AT, la influencia de la discapacidad de un hijo en la familia, y la relación entre las interacciones de los progenitores con los hijos y el desarrollo adecuado infantil.

En relación a la productividad, Ilama la atención que en la última década sólo se hallan encontrado 26 artículos de trabajo con familia contextualizados dentro de la AT en España. A pesar de la importancia del trabajo en familia en AT resulta escasa la literatura que se centra en investigar sus prácticas, y contracta con la cantidad de datos, evaluaciones, observaciones, diagnósticos, intervenciones, ... que diariamente se producen en un Centro de Atención e Intervención Temprana.

Es importante resaltar que la implementación de programas específicos que respondan a las necesidades de la familia y las investigaciones han ido avanzando gradualmente, tal y como observamos en la realidad de la práctica diaria en AT. No obstante, sigue siendo necesario contar con la implicación de los profesionales de la AT para progresar e implementar respuestas cada vez más personalizadas y eficaces adaptadas a las necesidades de las familias atendidas en AT. En este sentido, sería importante evaluar la cultura investigadora dentro de los equipos multidisciplinares en AT y promover distintas medidas dirigidas fundamentalmente a motivar la investigación entre los profesionales de AT, ya que continúa siendo una necesidad para aumentar los conocimientos sobre diferentes aspectos de la intervención. Tal y como indican Martínez Moreno y Calet. (2015) es necesario seguir indagando en:

a. Los agentes etiológicos y circunstancias psicosociales de los problemas que afectan a los niños/as.

b. Las repercusiones que las discapacidades o trastornos en el desarrollo tienen sobre la dinámica familiar.

c. Nuevos métodos de diagnóstico que permitan una detección más precoz de los problemas que posibilite una atención más temprana.

d. El desarrollo de nuevos programas de intervención más eficaces, conociendo qué formas de intervención conducen a la obtención de mejores resultados o qué aspectos concretos de cada forma de intervención hace que se produzcan resultados más satisfactorios. 


\section{ESTADO DEL ARTE DE LA INTERVENCIÓN CON FAMILIAS EN ATENCIÓN TEMPRANA DURANTE LA ÚLTIMA DÉCADA EN ESPAÑA}

Otros aspectos interesantes como futuras líneas temáticas podrían ser:

a. Indagar sobre qué aproximaciones específicas de intervención son más eficaces, profundizando en aqueIlos aspectos de la intervención que ayudan a conseguir mejores resultados, en qué características de los programas son más eficaces y en qué características de niño y de la familia contribuyen a la obtención de óptimos resultados.

b. Implementar estudios Iongitudinales y en entornos naturales que valoren la efectividad de la intervención a largo plazo y que midan su grado de generalización.

c. Desarrollar investigaciones interdisciplinares que permitan llevar a cabo un seguimiento a medio y largo plazo del desarrollo del niño.

d. Establecer un plan de difusión con los resultados de investigaciones actuales y recientes para que sean conocidos por los servicios y los distintos profesionales, y así poder evaluar las repercusiones que la investigación tiene en la práctica diaria y en la mejora de la calidad de los servicios.

e. Abrir una amplía línea de evaluación de los programas realizados dentro de la AT.

Por último, hay que destacar que la presente revisión bibliográfica ofrece una bondad principal: la replicabilidad. Más allá de los resultados presentados, este estudio muestra estrategias de análisis objetivas y fáciles de revisar, permitiendo la aparición de nuevos estudios que actualicen el conocimiento sobre la producción y contenidos en la literatura especializada en familia y AT.

\section{REFERENCIAS BIBLIOGRÁFICAS}

Alfonso, I. (1995). Técnicas de investigación bibliográfica. Caracas: Contextos Ediciones.

Brown, I., Galambos, D., Poston, D.J. y Turnbull, A.P. (2007). Person centered and family centered support. En I. Brown y M. Percy (Eds.), A comprehensive guide to intellectual and developmental disabilities (pp. 351-361). Baltimore: Paul H. Brookes.

Camarero, B.; Cuéllar-Flores, I.; Eimil, B.; Palacios, I.; Río, T. y del García-Villanova, F. (2012). La relación temprana en bebés prematuros. Intersubjetivo: Revista de Psicoterapia Psicoanalítica y Salud, 12, 2, 349-360.

Candel, I. (2005). Elaboración de un programa de Atención Temprana. Electronic Journal of Research in Educational Psychology, 3 (3), 151-192. D0l: http://dx.doi.org/10.25115/ejrep.v3i7.1206

Cañada Pérez, M.; Escorcia Mora, C.T.; Martínez Rico, G.; Alonso Martín, M.; Ayuso Blázquez, V.; Domenech Varea, M.; Franco Castellano, I.; Moltó Soler, E.; Roca Hurtuna, M. Santandreu García, A. y Sanchís Martínez, C. (2016). Atención temprana y prácticas centradas en la familia: A propósito de un caso. Revista de Logopedia, Foniatría y Audiología, 36, 185-194. D0I: https://doi.org/10.1016/j.rlfa.2016.07.004

Castellanos, P.; García-Sánchez, F.A.; Mendieta, P.; Gómez, L. y Rico, M.D. (2003). Intervención sobre las familias desde la figura de terapeuta-tutor del niño con necesidades de Atención Temprana. Siglo Cero, 34 (3), 5-18. Recuperado de https://sid.usal.es/idocs/F8/ART6708/articulos1.pdf

Clarissa Altina Cunha de Araújo, C.; Paz-Lourido, B. y Verger Gelabert, S. (2016). Tipos de apoyo a las familias con hijos con discapacidad y su influencia en la calidad de vida familiar. Ciência \& Saúde Coletiva, 21(10):3121-3130. DOl: https://doi.org/10.1590/1413-812320152110.18412016

Dalmau, M.; Balcells-Balcells, A.; Giné, C.; Cañadas, M.; Casas, 0.; Salat, Y.; Farré, V. y Calaf, N. (2017). Cómo implementar el modelo centrado en la familia en la intervención temprana. Anales de Psicología, 33 (3), 641651. D0l: http://dx.doi.org/10.6018/analesps.33.3.263611

de Andrés Viloria, C. y Guinea, C.L. (2012). La Atención a la Familia en Atención Temprana: Retos Actuales. Psicología Educativa, 18, 2, 123-133. D0l: http://dx.doi.org/10.5093/ed2012a13

Division for Early Childhood (2014). DEC recommended practices in early intervention/early childhood special education 2014. Recuperado de htpp://www.dec-sped.org/recommendedpractices. 
Dunst, C.J.; Johanson, C.; Trivette, C.M. y Hamby, D. (1991). Family-oriented early intervention policies and practices: Family-centered or not? Exceptional Children, 58, 115-126. DOl: https://doi.org/10.1177\%2F001440299105800203

Escorcia, C.T.; García-Sánchez, F.A.; Orcajada, N. y Sánchez-López, M.C. (2016). Perspectiva de las prácticas de Atencion Temprana centradas en la familia desde la logopedia. Revista de Logopedia, Foniatría y Audiología, 36 (4), 170-177. DOl: http://dx.doi.org/10.1016/j.rlfa.2016.07.002

Escorcia-Mora, C.T.; García-Sánchez, F.A., Sánchez-López, M.C., Orcajada, N. y Hernández-Pérez, E. (2018). Prácticas de Atención Temprana en el Sureste de España: Perspectiva de profesionales y familias. Anales de Psicología, 34 (3), 500-509. D0I: http://dx.doi.org/10.6018/analesps.34.3.311221

Espe-Sherwindt, M. (2008). Family-centred practice: Collaboration, competency, and evidence. Support for Learning, 23, 136-143. D0l: http://dx.doi.org/10.1111/j.1467-9604.2008.00384.x

Espe-Sherwindt, M. y Serrano, A.M. (2016). It takes two: The role of family-centered practices in communication intervention. Revista de Logopedia, Foniatría y Audiología, 36, 162-169. D0I: http://dx.doi.org/10.1016/j.rlfa.2016.07.006

Federación Estatal de Asociaciones de Profesionales de Atención Temprana (2008). Organización Diagnóstica para la Atención Temprana. Manual de Instrucciones. Madrid: Real Patronato sobre Discapacidad. Recuperado http://riberdis.cedd.net/bitstream/handle/11181/2978/Organizacion_diagnostica_atencion_temprana.pdf?s equence $=1 \&$ rd $=003164973331916$

Gracia, M.; Ausejo, R. y Porras, M. (2010). Intervención temprana en comunicación y lenguaje: Colaboración con las educadoras y familias de dos niños. Revista de Logopedia, Foniatría y Audiología, 30, 4, 186-195. D0I:10.1016/S0214-4603(10)70155-8

García, M.P.; Madrid, D. y Galante, R. (2017). Children and Augmentative or Alternative Communication System (AACs). A perceptive vision of the role played by families and professionals. Anales de Psicología, 33 (2), 334-341. DOI: https://doi.org/10.6018/analesps.33.2.267631

García-Sánchez, F.A. (2002). Reflexiones acerca del futuro de la Atención Temprana desde un modelo integral de intervención. Siglo Cero, 32 (2), 5-14. Recuperado de https://webs.um.es/fags/docs/2002sg0_futuro_at.pdf

García-Sánchez, F.A.; Escorcia, CT.; Sánchez-López, M.C.; Orcajada, N. y Hernández-Pérez, E. (2014). Atención temprana centrada en la familia. Siglo Cero, 45 (3), 6-27. Recuperado de http://www.desenvolupa.net/content/download/4934/43042/file/atencion_temprana_centrada_familia_v0.pdf

García-Sánchez, F.A.; Escorcia Mora, C.T. y Sánchez-López, M.C. (2014). Atención temprana centrada en la familia. Revista Española sobre Discapacidad Intelectual, 45 (3), 251, 6-27. Recuperado en http://www.desenvolupa.net/content/download/4934/43042/file/atencion_temprana_centrada_familia_v0.pdf

García-Sánchez, F.A.; Rubio-Gómez, N.; Orcajada-Sánchez, N.; Escorcia-Mora, C.T. y Cañadas, M. (2018). Necesidades de formación en prácticas centradas en la familia en profesionales de atención temprana españoles. Bordón, 70 (2), 39-55. DOl: https://doi.org/10.13042/Bordon.2018.59913

GAT (2005). Libro blanco de la Atención Temprana. Madrid: Real Patronato de Prevención y de Atención a Personas con Minusvalía. Recuperado de http://www.juntadeandalucia.es/salud/servicios/contenidos/andaluciaessalud/doc/LibroBlancoAtenci\%C2\%A6nTemprana.pdf

Gine, C.; Gracia, M.; Vilaseca, R. y García-Die, M.T. (2006). Repensar la Atención Temprana: Propuestas para un desarrollo futuro. Infancia y Aprendizaje, 29 (3), 297-313. DOl: https://doi.org/10.1174/021037006778147935

González Serrano, F. (2010). Nacer de nuevo: La crianza de los niños prematuros: Aspectos evolutivos. Atencion y acompañamiento al bebé y la familia. Cuadernos de Psiquiatría y Psicoterapia del niño y del adolescente, 49, 133-152. Recuperado en https://www.sepypna.com/documentos/articulos/nacer-crianza-ninos-prematuros.pdf 
Gútiez, P. (2005). Atención Temprana: prevención, detección e intervención en el desarrollo (0- 6 años) y sus alteraciones. Madrid: Editorial Complutense.

Gútiez, P. (2010). Early Childhood intervention in Spain: Standard needs and changes. International Journal of Early Childhood Special Education, 2 (2), 136-148. Recuperado de https://www.intjecse.net/index.php/ijecse/article/view/32/24

Icart Isern, M.T. y Canela Soler, J. (1994). El artículo de revisión. Enfermería Clínica, 4 (4), 180-184.

Lotka, A.J. (1926). The frequency distribution of scientific productivity. Journal of Washington Academy of Sciences, 16, 317-323.

Marco Arenas, M.; Sánchez López, M.C. y García Sánchez, F.A. (2018). Inteligencia emocional y prácticas relacionales con la familia en atención temprana. Siglo Cero, 49 (2), 266, 7-25. DOl: http://dx.doi.org/10.14201/scer02018492725

Martínez Moreno, A. y Calet, N. (2015). Intervención en Atención Temprana: Enfoque desde el ámbito familiar. Escritos de Psicología, 8, 2, 33-42. D0I: https://doi.org/ 10.5231/psy.writ.2015.1905 D0l: https://doi.org/ 10.5231/psy.writ.2015.1905

Mayorga-Fernández, M.J.; Madrid-Vivar, D. y García-Martínez, M.P. (2015). Aprender a trabajar con las familias en Atención Temprana: Estudio de caso. Escritos de Psicología, 8, 2, 52-60. D0I: https://doi.org/ 10.5231/psy.writ.2015.1306

Molina Vizcaíno, J.R. (2014). El uso psicoterapéutico del CBCL 11⁄2-5 con familias de niños/as atendidos en los centros de atención temprana. Cuadernos de Psiquiatría y Psicoterapia del Niño y del Adolescente, 58, 1724. Recuperado en https://www.sepypna.com/documentos/articulos/psiquiatria-58/04-jr-molina.pdf

Montagut Asunción, M.; D'Ocon Giménez, A. y Pastor Cerezuela, G. (2017). Interacción social y comunicación temprana en hermanos de niños con diagnóstico de trastorno del espectro autista: Una revisión de la literatura. Revista de Psicología, 1 (3), 373-388. D0I: https://doi.org/10.17060/ijodaep.2017.n1.v4.1065

Perea Velasco, L.P.; Martínez-Fuentes, M.T.; Pérez-López, J. y Díaz-Herrero, A. (2011). Interacción madre-hijo y desarrollo mental infantil: Implicaciones para la atención temprana. Revista de Psicología, 1, 1, 521-530. Recuperado en http://infad.eu/RevistalNFAD/2011/n1/volumen1/INFAD_010123_521-530.pdf

Pérez-López, J.; Pérez-Lag, M.; Montealegre Ramón, M.P. y Perea Velasco L. P. (2012). Estrés parental, desarrollo infantil y atención temprana. International Journal of Developmental and Educational Psychology, 1, 1, 123132. Recuperado en http://www.redalyc.org/articulo.oa?id=349832342012

Robles Bello, M.; Sánchez-Teruel, D. y Romero-Prado, A.B. (2015). Evaluación del estrés en padres de una niña con enfermedad rara en tratamiento en atención infantil temprana. Ansiedad y Estrés, 21 (1), 43-55. Recuperado en https://es.scribd.com/document/344155090/Evaluacion-Del-Estres-en-Padres-de-UnaNina-Con-Enfermedad-Rara-en-Tratamiento-en-Atencion-Infantil-Temprana

Roncallo, C.P.; Sánchez de Miguel, M. y Arranz Freijo, E.(2015). Vínculo materno-fetal: implicaciones en el desarrollo psicológico y propuesta de intervención en atención temprana. Escritos de Psicología, 8, 2, 14-23. DOI: https://doi.org/ 10.5231/psy.writ.2015.0706

Ruiz-Fernández, I.; Fajardo, I.; Bermejo, L.; Calzado, Z; Pérez-Ruiz, M.; García, V. y Gordillo, M. (2012). Atención temprana e intervención familiar. International Journal of Developmental and Educational Psychology, 3, 1, 39-47. Recuperado en http://www.redalyc.org/articulo.oa?id=349832338003

Sánchez, D. y Roble, M.A. (2015). Respuesta a un programa de resiliencia aplicado a padres de niños con Síndrome de Down. Universitas Psychologica, $14 \quad$ (2), 15-25. D0l: http://dx.doi.org.10.11144/Javeriana.upsy14-1.rpra

Sánchez Meca, J.(2010). Como realizar una revisión sistemática y un meta-análisis. Aula Abierta, 38, 2, 53-64. Recuperado de https://www.um.es/metaanalysis/pdf/5030.pdf

Torres Gómez, A. y Rabasot Solán, M.A. (2011). Aspectos emocionales en niños con discapacidad y sus familias. Experiencia en un centro de atención temprana. Revista de Psicoterapia Psicoanalítica y Salud, 11, 2, 331-348. 
UNESCO (2015). Investing against Evidence the Global State of Early Childhood Care and Education. Paris. Recuperado de https://unesdoc.unesco.org/ark:/48223/pf0000233558

Urrútia, G. y Bonfill, X. (2010). Declaración PRISMA: una propuesta para mejorar la publicación de revisiones sistemáticas y metaanálisis. Medicina Clínica, 135(11) 507-511. D0I: http://dx.doi. org/10.1016/j.medcli.2010.01.015

World Health Organization (2012). Early Childhood Development and Disability: A discussion paper. Geneva: World Health Organization. Recuperado de https://apps.who.int/iris/bitstream/handle/10665/75355/9789241504065_eng.pdf;jsessionid=4A794F58B4 B2FF1329B7D3EA19D65C5D?sequence=1 
